\title{
The Use of Botulinum ToXin In the Treatment of Headaches
}

\begin{abstract}
Alexander Mauskop, MD
Migraine is a disabling condition which affects 28 million Americans. Over 10 million Americans suffer from daily headaches. The discovery of triptans has revolutionized abortive therapy of migraines. However, patients suffering from frequent attacks and those who do not respond or do not tolerate triptans require prophylactic therapy. Many non-pharmacological treatments, such as biofeedback, magnesium and $\mathrm{CoQ}_{10}$ can be effective for some patients. Prophylactic pharmacotherapy of migraine headaches is limited in
\end{abstract}

its efficacy and has a potential for systemic side effects. The use of botulinum toxin for movement disorders and cosmesis led to an accidental discovery of its beneficial effect on headaches. Extensive anecdotal evidence accumulated over the past seven years and controlled trials suggest that intermittent and chronic migraines as well as chronic tensiontype headaches may respond to botulinum toxin injections. The effect of a single treatment, which is technically simple to administer, usually lasts three months. Botulinum toxin does not cause systemic or any other serious side effects. Many patients prefer botulinum toxin injections over prophylactic drugs because of its apparently high efficacy and remarkable safety. Several large controlled trials currently underway should lead to a wider acceptance of this treatment by neurologists and pain specialists.

Keywords: Botulinum toxin, headache, migraine, tension-type headache, headache management

\section{HISTORY}

Justinus Kerner (1) described the clinical picture of poisoning with botulinum toxin (BT) in spoiled sausage in 1817. (The word botulism is derived from the Latin botulus, which means sausage). Kerner (1) established that death from poisoning by BT was due to paralysis of muscles, and he was also the first to suggest possible therapeutic use for this toxin. A Belgian microbiologist, EmilePierre van Ermengem, first identified the bacterium, Clostridium botulinum, in 1895. Seven different serotypes of BT (A through $\mathrm{G}$ ) are produced by $C$ botulinum. Each of these serotypes produces muscle paralysis, but they vary with respect to the onset of action, duration of action, degree of diffusion in the tissues, and other properties. Only types A and B are available for clinical use.

In the 1970s, Alan Scott tested BT type A (BT-A), a product developed by Edward Schantz, in animals and humans. In 1989, the first commercial preparation of BT-A, Botox (Allergan, Irvine, CA) was

From New York Headache Center, New York, New York. Address Correspondence: Alexander Mauskop, MD, New York Headache Center, 30 East 76th Street, New York, NY 10021.

E-mail: NYheadache@aol.com

Funding: There was no external funding in preparation of this manuscript.

Conflict of Interest: None approved by the Food and Drug Administration (FDA) as an orphan drug for clinical use in blepharospasm and strabismus. Myobloc, BT type B (BT-B) was introduced in the US more recently, while outside the US a second BT-A product, Dysport is also available. Several serendipitous discoveries have led to a remarkable increase in the number of indications for BT.

\section{Mechanism of Action}

BT produces paralysis by disrupting the normal functioning of the neuromuscular junction. Presynaptic axon terminals release acetylcholine, a neurotransmitter that attaches to a postsynaptic receptor on the muscle and produces contraction of that muscle. After being injected into the muscle, BT enters the terminal part of the motor axon. Then it binds to the protein that is required for the fusion of acetylcholine-containing vesicles with the cell membrane, thus preventing the release of acetylcholine into the synaptic cleft. This effect occurs after an average of 4 to 6 days for BT-A and 2 to 4 days for BT type B (BT-B) and persists for an average of 3 months for BT-A and somewhat less for BT-B (2). This neuromuscular blockade is always reversible. Remarkably, even after years of repeated injections of BT into the same muscle, recovery of muscle function is always complete.

\section{Product Variation}

BT is a biological product. Different commercial products have distinct properties, and clinical effects may differ. Data obtained with one product should not be extrapolated to the others. For this reason, brand names will be used in this review.

A very small number of patients (less than 3\%) develop antibodies to BT after repeated injections. Botox has less protein than the other 2 commercially available products, Myobloc and Dysport, making it theoretically less likely to cause antibody formation. It appears that patients who stop responding to Botox because of antibody development may respond to Myobloc. This can be explained by the fact that different intracellular protein complexes needed to fuse vesicles to cell membranes are disrupted by different serotypes of BT.

The available BT preparations also differ in that Myobloc has a very acidic $\mathrm{pH}$ of 5.6, making injections more painful. Myobloc may diffuse more widely outside the injection area than Botox, which is suggested by higher incidence of dry mouth when it is injected into the neck muscles for torticollis.

Botox is available vacuum-dried in 100 -unit vials, while Myobloc is available in $0.5 \mathrm{ml}, 1 \mathrm{ml}$ and $2 \mathrm{ml}$ vials containing 5000 units per $\mathrm{ml}$. The units in both prep- 
arations are based on a lethal dose in mice, however different mouse assays were used for these two products, which accounts for the wide disparity in unit strength. There have been no trials comparing the efficacy of Botox and Myobloc, but anecdotal reports suggest that one unit of Botox is equivalent to 50 or possibly more units of Myobloc.

\section{Clinical Uses}

Treatment of blepharospasm was the first FDA-approved indication for the use of Botox. It was also approved for strabismus, but it is rarely used for this indication because of the relatively short duration of the effect and better alternatives for treatment. Soon after its introduction in 1989, Botox became widely used for cervical dystonias or torticollis, writer's cramp, spastic dysphonia and other dystonias. BT can also block salivation and perspiration by blocking release of acetylcholine, which is released by nerve endings to stimulate the action of the salivary and sweat glands.

Treatment of torticollis led to the observation that pain associated with this condition was also relieved (3), sometimes even when abnormal head position persisted. This led to its use for the treatment of pain of muscular origin, most commonly chronic lower and upper back pain as well as neck pain without torticollis. A double blind trial confirmed the efficacy of Botox in the treatment of chronic low back pain (4). Other uses of Botox include spasm of the lower esophageal sphincter, anal fissures, hyperhydrosis, excessive salivation and elimination of facial wrinkles. Both Botox and Myobloc are now approved for cervical dystonia, while Botox is also approved for disorders of the facial nerve, such as hemifacial spasm as well as strabismus and glabellar lines. FDA approval of Botox for hyperhydrosis is expected in the near future.

\section{Safety and Tolerability}

The extensive experience with Botox in a large number of patients over long periods of time has demonstrated the remarkable safety of this toxin. No serious allergic reactions have ever been reported with Botox, although rarely rash and flulike symptoms can occur. Injection of anterior neck muscles can cause swallowing difficulties in a small number of patients; nasogastric tube feeding has been required in a few reported patients. Diffi- culty swallowing and dry mouth appear to be more common with injections of Myobloc because of its wider diffusion. The most common side effects when treating facial muscles are cosmetic. These include ptosis or asymmetry of the position of the eyebrows and they usually resolve over a period of several weeks. In a rare patient they may last for up to 2 to 3 months. Additional BT injections can correct asymmetry and even ptosis. Headache patients occasionally develop a headache following the procedure, although in some, immediate relief of an acute attack can also occur. The latter is most likely due to trigger point injection effect. Difficulty in holding the head erect because of neck muscle weakness is another possible, but rare side effect. Worsening of headaches and muscle pain can occur for several days after the injections because of the delay in the muscle relaxing effect of BT.

\section{BT in the Management of Headaches}

Widespread use of Botox for the treatment of forehead wrinkles led to the observation by a plastic surgeon, William Binder, as well as physicians administering Botox for cranial movement disorders, that many patients reported relief of their migraine headaches (5). These and subsequent observations and studies challenged our understanding of migraine pathogenesis.

\section{Mechanism of Action}

The possible mechanism of action by which BT prevents migraine headaches is unclear. Migraine is a brain disorder, and PET scanning has identified a "migraine generator" in the brainstem where migraine attacks begin. The most logical explanation for the efficacy of BT is that in order for migraine pain to start, pericranial muscles must contract and send afferent input to the brainstem. BT reduces both efferent input from the nerve to the muscle as well as afferent activity from the muscle into the central nervous system (6). It is possible that relaxation of pericranial muscles reduces afferent input into the brainstem migraine generator, thus raising the threshold for a migraine attack.

This theory is supported by the author's observation of patients with migraine with aura who, after treatment with BT, report relief of their migraine headache and all associated symptoms but continue to experience visual aura.
Another possibility is that BT is transported along the axons into the brainstem and exerts its effect centrally (7). This appears less likely, since the author observed in many patients a close correlation between the return of muscle strength and the return of headaches. BT has also been found to inhibit the release of substance $P$ from trigeminal nerve endings, although the role of substance $\mathrm{P}$ in the pathogenesis of migraines is not clear. Theoretically tension-type headaches should be more amenable to treatment with Botox than migraines, although the author's experience and published reports suggest otherwise. This observation may be explained by electromyographic (EMG) findings that show more pronounced pericranial muscle spasm in patients having a migraine attack than in patients having a tension headache.

\section{TRIALS OF BT IN HEADACHE}

Many anecdotal reports and several small, double-blind studies suggest that Botox is useful for a variety of headache syndromes $(8,9)$ including migraine $(5,8$, 10-14), tension-type $(9,15-20)$, cervicogenic $(21,22)$, chronic daily (23-25) and even cluster headaches $(26,27)$. Discussion of the most salient studies follows.

\section{Published Studies of Botulinum Toxin Type A in Migraine Headache}

Silberstein et al (28) assessed the safety and efficacy of Botox in the prevention of migraine in a double-blind, vehicle-controlled study with outcomes that included changes in the frequency and severity of migraines, migraine-associated symptoms, and days of medication use for acute migraine. The $25-\mathrm{U}$ and $75-\mathrm{U}$ doses were studied. Compared with patients in the vehicle-placebo group, those in the 25-U group had significantly fewer migraine attacks per month, less severe migraines, fewer days when they needed acute migraine medication, and less migraine-associated vomiting. Global assessments were significantly better in the $25-\mathrm{U}$ and $75-\mathrm{U}$ groups than in the placebo group, but the $75-\mathrm{U}$ group experienced more treatment-related adverse events. The investigators concluded that Botox, $25 \mathrm{U}$, was safe and reduced migraine frequency, severity, acute medication use, and migraine-associated vomiting. The $25-\mathrm{U}$ dose, but not the $75-\mathrm{U}$ dose was found to be more effective than placebo. The trial design did not take into account 
the great variability of migraine pain distribution. The injections were given only into the forehead and temples, while many migraine patients report predominantly occipital or temporoparietal distribution of pain. It can be speculated that patients in the 25-U group had predominantly frontal pain, while those in the 75$\mathrm{U}$ group did not. A therapeutic window effect where a higher dose is less effective is a less plausible explanation.

In a nonrandomized, open-label trial conducted in 106 patients, Binder et al tested the ability of Botox to alleviate migraine headache frequency and severity (5). Of the group, 77 participants were judged to have true migraine, and $51 \%$ of these reported complete response with a mean duration of response of 4.1 months. Of the 10 patients with true migraine who were treated during the acute phase of an attack, $70 \%$ reported a complete response within 1 to 2 hours.

Blumenfeld et al (29) studied the impact of BT-A treatment on medication costs and usage in difficult-to- treat chronic headache in a retrospective review of 5 case studies, with the study outcome being change in the annual cost of headache-related medication usage. The patients had migraine that had been unresponsive to conventional therapy. All but one also had experienced features that were typical of tension-type, transformed, chronic daily, and/or rebound headache. Botox was administered in doses of 40 to $100 \mathrm{U}$ at multiple sites corresponding to the pain patterns. Improvements lasted up to 2 months, and there were decreases in migraine frequency, severity, and medication costs. No adverse events were reported in this group.

The impact of Botox on quality of life and triptan use was assessed by Relja (30) in 32 patients enrolled in a doubleblind, randomized, placebo controlled trial. All patients were determined to have moderate or severe migraine-related disability according to MIDAS. Treatments included Botox, $100 \mathrm{U}$, or placebo. Botox reduced the impact of migraine on normal daily activities, reduced requirements for additional medication, reduced total triptan dosage and use, and reduced pain characteristics. No change in the number of total headache-free days was noted. The change in pain characteristics was described as a shift from migraine-type pain to the pain typically associated with easier-to-tolerate tension-type headaches.
The use of BT-A in patients with chronic daily headaches associated with migraine was evaluated by Klapper and Klapper (24) in an open-label, prospective study of 5 patients. BT was administered at multiple and fixed sites and at a fixed, 75-U dose. Four of the 5 patients experienced marked improvements in their tension-type and migraine headaches. No adverse events were reported.

A single case report by Poungvarin (31) in 2002 of Botox use in a patient with status migrainous suggests a benefit for patients in this situation, as well. Botox was injected in a single $25-\mathrm{U}$ dose at a single site. Symptoms improved within 1 hour, and status migrainous was aborted within 10 hours. Headaches were completely eliminated for 2 months after treatment. The authors termed the improvement "dramatic."

\section{Abstracts of Botulinum Toxin Type A in Migraine Headache}

In 2003, Blumenfeld (32) reported on the efficacy of BT-A in reducing headache frequency and intensity in a retrospective, open-label analysis that included 271 patients. All participants had disabling, chronic migraine. BT-A was administered at an average, fixed dose of 63.2 $\mathrm{U}$ either at a fixed site or in a pattern that followed the pain. A response to treatment was reported by $80 \%$ of patients, and significant reductions in the frequency of headache occurred (from 18.9 to 8.3 days/month). Headache intensity was diminished by $25 \%$. The author concluded that BT-A provides "efficacious and safe" preventive treatment for headaches.

Mathew et al (33) reported on their long-term experience with BT-A in patients with chronic headache in a retrospective, open-label trial of 208 patients. All participants had disabling, chronic migraine and were treated with 50 to 100 $\mathrm{U}$ of the study medication administered at fixed sites or at sites that corresponded to the location of pain. According to the physician's global assessment, the 100 $\mathrm{U}$ dose was more effective than the $50-\mathrm{U}$ dose. The incidence of severe, disabling migraine was greatly reduced compared with the incidence of less severe headache. Patients returned for treatment as the effects of an injection wore off, and there was no evidence of tachyphylaxis. This observation also suggests that the benefits were not attributable to a placebo ef- fect. Botox therapy significantly reduced the disability associated with migraine, as well as migraine frequency and the need for other, acute medications. No patient dropped out of the study because of a lack of efficacy.

A randomized, double-blind, placebo-controlled trial enrolling 30 patients was reported by Barrientos et al (34) in 2002. Using a follow the pain approach, $50 \mathrm{U}$ of BT-A was administered. The frequency, duration, acute use of migraine medications, and the incidence of migraine with nausea were decreased among patients receiving the study medication. Improvements lasted up to 3 months. One patient experienced frontal asymmetry that lasted for 30 days. The authors concluded that BT-A provided effective treatment of migraine in this patient group and that it warrants additional study.

A prospective, open-label disability study was reported by Eross et al (35) in 2002. Fifty-four patients completed the study, which investigated the effects of BT-A on disability in patients suffering from chronic and episodic migraine. The dosage of study drug ranged from 25 to $75 \mathrm{U}$; it was administered at multiple sites. Improvements lasted up to 3 months, the frequency of headaches was diminished, and disability was lessened.

The efficacy of BT-A for the treatment of patients with cervicogenic migraine was studied in a prospective, openlabel by Krusz (36) in 2002. The study dose was $100 \mathrm{U}$ administered at 4 to 6 posterior cervical injection sites. Headache frequency was reduced by more than $70 \%$, and migraine severity was judged to be diminished by $50 \%$. The investigators concluded that BT-A was effective in reducing headache and spasm symptoms in this patient population.

This author evaluated the long-term efficacy of Botox in the treatment of episodic and chronic migraine headaches in a retrospective, open-label trial in patients treated multiple times and over long periods of time (12). In some, multiple other treatments had failed. Dosages of 25 to $200 \mathrm{U}$ were administered in a follow-the-pain pattern. Among the findings were that improvements lasted up to 15 months, headaches were completely eliminated in some patients, and symptom relief from abortive drugs was improved by Botox treatment. In addition, headache frequency and intensity, as well 
as triptan use, were diminished. Adverse events included transient neck pain and weakness in 2 patients, acute headache in 2 patients, and neck weakness and a fainting feeling in 1 patient each. At the time of this review the author has treated over 900 headache patients with Botox in clinical practice.

Abstracts and Published Articles of Botulinum Toxin Type A in TensionType Headache, Chronic Migraine, Chronic Daily Headache and Other HEADACHE TYPES

BT has also been the subject of numerous investigations conducted in patients with tension-type headaches, chronic daily headache, and other forms of headache. In 1999, Smuts et al (37) assessed the efficacy of Botox in the prophylaxis of chronic tension-type headaches. This double-blind, randomized, placebocontrolled study enrolled 37 patients, and outcomes included changes in headache intensity, headache-free days, and chronic pain index. Patients were randomized to receive $100 \mathrm{U}$ of Botox or placebo. The number of headache-free days improved significantly in the Botox group relative to the placebo group, and patients randomized to Botox reported improvement in quality of life after the injections. Improvements lasted for 3 months, and no serious side effects were reported.

A similar double-blind, placebo controlled trail of BT-A was conducted in 16 patients by Relja (18). The study agent was administered in doses of 35 to $80 \mathrm{U}$ in a follow the pain approach and at multiple sites. The conclusion was that BTA provided safe and effective prophylaxis of tension-type headache. Improvement lasted for up to 2 months, and no adverse events were reported.

In a study of chronic cervical-associated headache associated with whiplash injuries, Freund et al (38) conducted a randomized, double-blind, placebo-controlled trail in 26 patients who received BT-A at a dose of $100 \mathrm{U}$ or placebo. Pain was diminished and cervical range of motion increased, but the authors made no conclusions about efficacy because of the short follow-up and small sample size.

Porta (39) compared the efficacy of Botox with that of methylprednisolone in a randomized, single-blind trial conducted in 20 patients with tension-type headaches. The test regimens were Botox, 5 to $15 \mathrm{U}$ in lidocaine versus methylpredniso- lone, $40 \mathrm{mg}$ plus lidocaine. The corticosteroid decreased pain at 30 days, while Botox was associated with diminished pain at 30 days and 60 days. Botox was also associated with improvements in the symptom profile for up to 60 days. No adverse events were reported. According to the authors, the results confirmed the effectiveness of Botox for the treatment of tension headache and Botox produced more prolonged pain relief than methylprednisolone.

Among the published, peer-reviewed abstracts on this subjects is that of McAllister (40), who reported on improvements in headache and changes in headache medications among 116 patients with a variety of headache types (migraine with or without aura, episodic tension-type headache, cervicogenic headache, and cervical myofascial pain) who were treated with Botox in a retrospective, open-label analysis. Injections were administered both at fixed sites and in a follow the pain pattern, and dosages ranged from 40 to $280 \mathrm{U}$. Of note, all patients reported some degree of headache improvement, with $76 \%$ reporting an improvement of $75 \%$ or more; $9 \%$ reported a complete remission of headache. The monthly cost of headache medications decreased from $\$ 253$ to $\$ 97$. The investigators called for larger trials to confirm these results.

Using the Migraine Disability Assessment Scale (MIDAS), Tepper et al (41) assessed the effect of Botox on the disability caused by refractory headache in $100 \mathrm{pa}$ tients included in a retrospective, open-label trial. Participants in this trial had previous failures of at least 4 preventive treatments. The headache types represented in this group included chronic daily headache with analgesic rebound headache, chronic daily headache without analgesic rebound headache, and chronic, posttraumatic headache. Botox was administered in a dose of $25 \mathrm{U}$. The mean MIDAS score dropped from 34.5 to 15.9 , a difference that was statistically significant at the level of $P<0.001$.

The efficacy of Botox in patients with chronic, intractable headache with or without concomitant neck pain was the subject of a study by Miller et al (42), who conducted a prospective, open-label study in 68 patients. All subjects had unsuccessful trials with other treatment modalities. Botox was administered at a dosage of $100 \mathrm{U}$ in a follow the pain proto- col. A total of $75 \%$ of patients reported $50 \%$ to $100 \%$ pain relief. A total of $13 \%$ reported no benefit, and $12 \%$ judged their improvements to be of little clinical use. Treatment efficacy was similar in patients with and without neck pain.

Ten adolescent females were the subjects of a study by Tomosovic et al (43). All subjects had chronic daily headaches that had been refractory to other modalities. Symptom improvements were assessed with MIDAS. Botox, $100 \mathrm{U}$, was administered in a follow the pain approach. All participants reported improvement, and 8 of the 10 had sustained improvement at 90 days. The use of other medications was reduced, as well.

Stiff person syndrome was the subject of a study by Loder (44) in 2003. This case report described the response to Botox in a patient with stiff person syndrome accompanied by headache and neck pain. Botox, $100 \mathrm{U}$, was administered. At 3 weeks postinjection, the patient reported complete resolution of the headache. Muscle relaxation was credited as the most likely mechanism of action, although other mechanisms were considered possible.

Troost (45) studied the impact of repeated Botox treatments in 436 patients with intractable migraine or episodic tension-type headaches. Botox dosages were in the range of 25 to $300 \mathrm{U}$ and were given at fixed and multiple sites. A total of $91 \%$ of patients reported improvements, and the more cycles of treatment a patient had, the greater the improvements. Improvements were cumulative through 3 cycles of treatment and sustained through 8 treatments. Minor injection-site pain was the only adverse reaction reported in this series. Importantly, tachyphylaxis was not observed.

In 1998, Wheeler (46) published 4 case reports of the use of BT-A as adjunctive therapy for refractory headache associated with pericranial muscle tension. The medication was injected at multiple sites corresponding to the pain patterns. The frequency and severity of headaches diminished, as did the need for subsequent medical and physical therapy interventions.

In contrast, Sebastian et al (47) were unable to show a benefit for Botox in their 12-week, double-blind, placebo-controlled trial in 40 patients with chronic, tension-type headache. Subjects were treated with $100 \mathrm{U}$ of Botox or place- 
bo. No significant difference between the groups was apparent with respect to average headache days, headache hours each day, requirements for additional symptom management, or the patient global assessments.

Blumenfeld (48) assessed the efficacy of Botox as prophylactic therapy in 271 patients with a range of headache types, including chronic daily, episodic tension, episodic migraine, or mixed headache. Mean Botox dose was $63.2 \mathrm{U}$ administered at multiple sites. Headache intensity and frequency was diminished by treatment, and improvements persisted for more than 8 months. Three patients experienced transient ptosis.

To evaluate the possibility that Botox is associated with progressive and $\mathrm{cu}-$ mulative treatment effects, Troost (49) administered the drug in doses of 30 to 240 $\mathrm{U}$ to 134 patients enrolled in a prospective, open label study. The subjects represented an array of headache types. No adverse events were reported, but pain was diminished. Headache scores improved according to both patient and clinical ratings. Improvements again persisted beyond 8 months.

Fifty-six patients with chronic daily headache were enrolled in Klapper's $(23,25)$ double-blind, placebo-controlled study of Botox for the prophylaxis of chronic daily headache. Treatment groups included Botox $100 \mathrm{U}$, Botox 27.5 U plus placebo, Botox $72.5 \mathrm{U}$ plus placebo, or placebo alone. Both headache duration and frequency were reduced by active treatment.

Padberg et al (50) reported nonsignificant improvements in headache intensity and frequency, headache-free days, and medication days among 40 patients with chronic, tension-type headaches who were enrolled in a randomized, double-blind, placebo-controlled study. The Botox dosage was $100 \mathrm{U}$ administered at multiple, individualized sites. Improvements persisted for up to 3 months.

Sixty patients with chronic daily headache were enrolled in Ondo's (51) double-blind, placebo-controlled trial that employed a Botox dose of $200 \mathrm{U}$ or placebo in a follow the pain approach. Based on improvements in headachefree days, frequency, the need for abortive medications, and global impressions that persisted for up to 3 months, the authors concluded that Botox was a potentially helpful approach to this form of headache and that its effects appeared to be cumulative.

Smuts (10) investigated the efficacy of Botox in 79 patients with a variety of headache types enrolled in a prospective, open-label trial of Botox, $100 \mathrm{U}$. Positive outcomes were reported in $50 \%$ to $68 \%$ of patients with chronic tension-type headaches, migraine, cluster headache, and cervicogenic headaches. The authors concluded that Botox could be considered an alternative therapy in patients with a variety of refractory headache syndromes.

Improvements lasting longer than 3 months and decreases in pain frequency, intensity, tenderness and duration of pain were among the findings of Lopez-Lozano (52), who studied Botox in 10 patients with chronic tension-type headaches accompanied by pericranial muscle tenderness. The dose of Botox was unspecified, and a single treatment at a single site was administered. Only the improvement in duration of pain was nonsignificant, and the authors concluded that Botox had produced "significant" improvements in patients with chronic tension-type headache associated with pericranial muscle tension.

A retrospective case review of 10 patients also with chronic tension-type headache was reported by Lin and Chang (53) in 2002. Botox was administered at doses of $20 \mathrm{U}$ to $100 \mathrm{U}$ at multiple sites and for multiple treatments. Pain severity and disability were diminished, and patients reported pain relief. The improvements persisted for more than 3.5 months. The authors took this as confirmation of the clinical benefit of Botox in treating this type of headache.

Miller and Denny (54) conducted a retrospective cohort analysis in 48 patients with chronic headache who were treated with Botox in a combination fixed injection and follow-the-pain protocol. The patients had not obtained adequate headache relief from other therapies. All received multiple treatments of 50 to 300 $\mathrm{U}$ of Botox. The response to Botox was rated as good, very good, or full in $71 \%$ of participants; $8 \%$ reported being headache-free after treatment. These investigators concluded that Botox therapy may be beneficial in patients who have not benefited from other headache therapies and that multiple regimens may be more effective than single treatments.

Twenty patients with chronic daily headache refractory to other treatment modalities were enrolled in a prospective, open-label study conducted by Edwards (55) in 2002. Botox was administered in dosages of 20 to $100 \mathrm{U}$. The mean headache frequency dropped from 7 to 3.5 days per week. No clinical weakness was observed, and the side effects were limited to injection site discomfort. The authors considered the benefits of Botox to be "highly significant." They noted that Botox might represent an alternative treatment for patients with chronic daily headache that poses no risk of drug abuse, drug-drug interaction, sedating effects, or other systemic toxicities.

The useful of Botox as prophylaxis for chronic tension-type headache was examined by Relja et al (19) in a 10month randomized, double-blind, placebo-controlled phase and in an 18-month, prospective, open-label phase. Thirty patients were enrolled. Botox was administered at doses of 40 to $95 \mathrm{U}$ in a followthe-pain pattern at multiple sites. During the placebo-controlled phase, the number of headache-free days was increased, while headache severity was diminished. During the open label period, tenderness diminished. Adverse events were rare.

Freund and Schwartz (56) reported on an open-label, prospective investigation of 8 patients with whiplash-associated neck pain who were treated with Botox. Botox doses were $100 \mathrm{U}$ or less. Pain was diminished, and range-of-motion improved. The investigators termed the acute improvements "profound" and called for blinded, placebo-controlled trials so that the efficacy of the agent in chronic whiplash can pain can be assessed.

Wollina (57) published the cases of 8 patients who reported that their tension headaches were relieved after they received Botox for cosmetic purposes (wrinkles). Johnstone et al (58) reported on one patient who received Botox for relief of facial pain, headache and blepharospasm. The improvements associated with a $27.5 \mathrm{U}$ dose at multiple locations lasted for 3 months. Headaches were relieved, and pain and blepharospasm were diminished.

\section{Review Articles: The Application of Botulinum Toxin Type-A in Headache}

At least 5 review articles on the use of BTX-A in patients with headache have been published to date. Silberstein (59) suggested that before BTX-A could be 
considered a first-line agent for the treatment of migraine, larger studies would be required to determine the optimal dosing and administration sites. This author published a review in 2002 stating that BTX-A is the preferred agent for many patients with migraine, since prophylactic pharmacotherapy of migraine has limited efficacy and because of the possibility of systemic side effects (12). In contrast, BTX-A is simple to administer, has few systemic effects, and the beneficial effects of a single dose may last for 3 months. In their summary of the numerous clinical uses for which BTX-A has been reported, Royal et al (60) noted that major benefit of BTX-A may be its duration of efficacy. Nonetheless, BTX-A was not recommended for first-line use. Instead, they suggested reserving its use for patients whose headaches are refractory to other modalities. Mathew et al advised clinicians to consider using BTX-A in patients with refractory forms of chronic migraine and chronic tension-type headaches, noting that the agent reduces the frequency and severity of headaches, improves disability scores and quality of life, and reduces the need for acute medication, all without the development of tachyphylaxis to repeated doses (61). A thorough review by Gobel et al (8) stressed excellent tolerability, safety and efficacy of BT-A in the treatment of headache syndromes and its advantages over pharmacotherapy.

\section{Treatment of Migraines}

\section{Nonpharmacologic Management}

In addition to trigger avoidance, mind-body techniques and alternative and complementary methods may help improve migraine symptoms. Patients need to understand the benefits and potential problems associated with these methods.

\section{Trigger Avoidance}

Migraine has been shown to be a genetic disorder. Genetic predisposition can usually be modified by a variety of triggering factors, however. As a result, treatment begins by educating the patient to avoid potential triggers such as stress, lack of sleep, not eating for prolonged periods, dehydration, and consumption of certain foods.

The best antidote for stress is regular aerobic exercise. For many patients, 20 to 30 minutes of vigorous exercise is sufficient if performed 4 to 5 times a week. Dietary changes occasionally lead to complete resolution of migraine symptoms, but in many patients they simply reduce the frequency of attacks. Foods that can cause migraine headaches in susceptible patients include chocolate, yogurt, bananas, dried fruit, beans, aged cheese, citrus fruit or juice, pickled and marinated foods, and buttermilk. Monosodium glutamate and aspartame should be avoided. Among the alcoholic beverages, red wine and beer are more likely to induce a migraine headache than vodka.

\section{Mind-Body Techniques}

Biofeedback, meditation and other relaxation techniques help prevent migraine headaches in many patients (6264). Biofeedback is a computerized relaxation technique that can be learned in 6 to 8 weekly sessions. Children are particularly adept at biofeedback and can master it in as few as 3 to 4 sessions.

\section{Nutritional Supplements and Herbal Remedies}

Magnesium can help prevent migraine headaches in up to $50 \%$ of patients. We gave an intravenous infusion of $1 \mathrm{~g}$ of magnesium sulfate to 40 consecutive patients with acute migraine (65). Of these 40 patients, 21 had very good and sustained relief of their headaches. Of those who responded, $86 \%$ had low serum ionized magnesium levels. Of the non-responders, only $16 \%$ had low values. Intravenous administration of magnesium in the treatment of cluster headaches leads to a $40 \%$ response rate. A correlation between clinical response and serum ionized magnesium levels was also found. Oral magnesium supplementation was used as prophylactic therapy for migraines in four double-blind trials. Two of the three trials $(66,67)$ in adults had positive findings, and one was negative. The negative study (68) used what turned out to be a poorly absorbed salt of magnesium, as indicated by the fact that almost half of the patients in the active group developed diarrhea. Magnesium oxide and magnesium diglycinate, in doses of 400 to $600 \mathrm{mg}$ are inexpensive and can be recommended routinely to all migraine sufferers. Slow-release magnesium chloride is an alternative if patients experienced diarrhea or other GI side effects in response to other magnesium products.

\section{Riboflavin}

Also called vitamin B-2, riboflavin reportedly relieved migraine headaches better than placebo in a double-blind study (69). The maximum effect was achieved after 3 months of a daily dosage of $400 \mathrm{mg}$.

\section{Co-enzyme $Q_{10}$}

A double-blind study (70) suggests that this co-enzyme can prevent headaches at a daily dosage of $300 \mathrm{mg}$. The results of a double-blind study of its efficacy in patients with Parkinson's disease at a dosage of $1200 \mathrm{mg} / \mathrm{d}$ suggest that it may have a beneficial effect on at least some aspects of brain function.

\section{Feverfew}

The only herbal remedy that has been submitted to multiple, well-designed double-blind trials (71-73), feverfew tends to provide better migraine prophylaxis than placebo. Because feverfew is fairly safe and may be helpful some patients, it is the herb to recommend to patients interested in herbal remedies.

\section{Butterbur Root}

Two double-blind studies $(74,75)$ of the extract of butterbur root have shown benefit in migraine patients. This plant and its root contain carcinogens, and homemade preparations can be dangerous. Only a commercial product made in Germany, Petadolex, is safe to use.

\section{Combination Products}

Several products combining a few different supplements and herbal remedies are now available. A combination of magnesium, riboflavin and feverfew (MigreLief, MigraHealth) is a convenient and expeditious way to try 3 safe and effective nonpharmacological products. It is possible, but not proven, that these agents have synergistic beneficial effects.

\section{Acupuncture}

The available data suggest that acupuncture has an analgesic action in patients with migraine. Animal studies indicate that this effect is mediated by 2 possible mechanisms: a naloxone-reversible endorphin mechanism and/or a serotonin-mediated process that is not reversed by naloxone (76). A review of 14 trials comparing true and sham acupuncture showed at least a trend in favor of acupuncture (77). 


\section{Pharmacologic Treatment}

Pharmacological therapy of migraines is divided into abortive treatment of individual attacks and daily prophylactic therapies. Aggressive treatment of individual attacks with migraine-specific drugs, such as triptans could possibly prevent the development of chronic migraines. Chronic daily headaches afflict $5 \%$ of the population.

\section{Non-specific abortive therapies}

Non-steroidal anti-inflammatory agents (NSAIDs) can be effective for milder migraine headaches. A more rapid onset can be achieved by using an effervescent form of aspirin (Alka-Seltzer) or solubilized ibuprofen (Advil Migraine, Advil Liquigel).

The combination of butalbital, caffeine, and either acetaminophen or aspirin can be somewhat helpful in some patients with migraine headaches. These are not highly effective agents, however, and they have possible side effects, including rebound headaches and addiction. Because of its potential for abuse and side effects and its lack of efficacy, butalbital was removed from the market in Germany.

A sympathomimetic amine with vasoconstrictive properties, $\mathrm{d}$ isometheptene, is available in combination with dichloralphenazone, a mild sedative, and acetaminophen (Midrin, Isocom, Duradrin). This combination can be effective in many patients who do not respond to other drugs. Drowsiness is a potential side effect. To avoid rebound headaches, patients should take no more than 20 of these tablets a month. The addition of codeine to some of the combinations (Fiorinal with codeine) improves their efficacy for severe headaches.

If a headache is accompanied by nausea, prochlorperazine (Compazine) via injection, tablet or suppository may be effective. Alternately, a tablet or injection of metoclopramide (Reglan) is often effective. A trial of other drugs in this category, including promethazine (Phenergan), trimethobenzamide (Tigan), or ondansetron (Zofran) may be appropriate, since they may have different efficacy and side effect profiles in specific patients.

A prolonged or refractory headache that does not respond to triptans or other drugs may resolve in response to a single large dose of corticosteroids, such as dexamethasone (Decadron), 6 to $8 \mathrm{mg}$ given orally or intravenously. A methyl- prednisolone (Medrol) dose pack is another option.

Opioid drugs seem to be less effective for migraine headaches than for other pain syndromes, mostly because side effects, such as drowsiness and nausea, appear to precede or accompany pain relief. As a result, opioids may relieve pain, but often do not improve a patient's functional status.

\section{Migraine-specific abortive therapies.}

Oral or rectal ergots can be very effective, but often cause nausea and other side effects. Dihydroergotamine can be given by several routes of administration, including the SC, IM, and IV (DHE45) routes and, with significantly lower efficacy, intranasally (Migranal). A parenteral dose of $1 \mathrm{mg}$ is sufficient for most patients, but some may require 2 or $3 \mathrm{mg}$. The starting dose is $0.5 \mathrm{mg}$ repeated in 45 minutes if necessary.

Sumatriptan (Imitrex) and other triptans were specifically developed to bind to $5 \mathrm{HT}_{1 \mathrm{~B}}$ and $5 \mathrm{HT}_{1 \mathrm{D}}$ serotonin receptor subtypes, which are involved in the pathogenesis of migraine headaches. The $5 \mathrm{HT}_{1 \mathrm{~B}}$ receptors are present predominantly in the cerebral blood vessels; very few are present in coronary blood vessels. In contrast, $5 \mathrm{HT}_{1 \mathrm{D}}$ receptors are found in neurons. Triptans relieve the pain, photophobia, phonophobia, and nausea associated with migraine and often return the patient to normal functioning. Sumatriptan is available in an injection (which can be self-administered), tablets, and nasal spray. Side effects are more common with the injected form and include a flushed sensation, non-cardiac chest pressure, paresthesias, and injection site pain. Other drugs in this class include rizatrip$\tan$ (Maxalt), almotriptan (Axert), zolmitriptan (Zomig), naratriptan (Amerge), eletriptan (Relpax) and frovatriptan (Frova).

The efficacy and side effect profiles of these drugs may differ in individual patients (78). For example, rizatriptan may have very good efficacy with a faster onset of action, while naratriptan may offer longer duration of action, but a slower onset. Almotriptan may be least likely to cause chest pressure while offering good efficacy. Zolmitriptan may produce a more consistent response. Sumatriptan nasal spray exhibits a very poor consistency of response from attack to attack in the same patient. These characterizations apply to most patients taking these drugs, but individual variations are often present. Patients may respond very well to one, but not another triptan or the slower acting one, such as naratriptan, may be quickest acting for a particular patient.

Side effects also vary greatly among patients. As a result, patients should be given trials of various triptans in the hope of finding one with rapid onset, complete relief, no side effects, and no symptom recurrence. Zolmitriptan and rizatriptan are available in tablet formulation and in orally disintegrating tablets, with latter offering the convenience of not having to take the medication with water.

The triptans contain a very small amount of aspartame, which in a rare patient can worsen the headache. Zolmitriptan is also available in nasal spray, which offers good efficacy with rapid onset of action and more consistent responses than sumatriptan nasal spray.

Sumatriptan and other triptans are contraindicated in patients with uncontrolled hypertension, ischemic heart disease, multiple risk factors for coronary artery disease, and complicated migraines (migraines that are accompanied by a transient neurological deficit). Complicated migraine, which is a rare condition, must be differentiated from migraine with sensory or motor aura. In the latter, neurological symptoms precede the headache and triptans are not contraindicated. In complicated migraine, neurological symptoms persist into the headache phase. This distinction may not be as important as the triptan package inserts suggest, however. Triptans have reportedly been used safely in patients with complicated migraine, probably because these symptoms are more likely to be caused by neuronal inhibition than vasoconstriction, which follows neuronal inhibition. Different triptans are not supposed to be given within 24 hours of each other, although no good theoretical or experiential explanation for this prohibition exists.

\section{Treatment of a Severe or Prolonged Attack}

A very severe or prolonged migraine episode can be treated with injectable medications much more efficiently in the office than in the emergency department (ED). Intravenous magnesium (1 g magnesium sulfate/10 cc normal saline, given by slow push over 5 minutes) provides 
prompt and complete relief in up to 50\% of patients. If the patient does not experience relief within 10 to 15 minutes, administer injectable sumatriptan followed by an IV push of 30 to $60 \mathrm{mg}$ of ketorolac (Toradol), This is followed in $15 \mathrm{~min}$ by an IV push of 6 to $8 \mathrm{mg}$ of dexamethasone and an IV push of 500 to $1000 \mathrm{mg}$ of valproic acid (Depakene). Unlike opioid drugs, which are commonly given in the ED, none of the drugs in this protocol is sedating, and all are much more effective than opioids. If nausea is not relieved by these measures, consider IV metoclopramide, $10 \mathrm{mg}$ or prochlorperazine (Compazine), $10 \mathrm{mg}$. Both of these agents may have some sedating effect.

\section{Prophylactic Therapy}

Tricyclic antidepressants (TCAs), as well as other antidepressants, can be effective for migraine headaches, but their potential to cause weight gain deters many young women who suffer from migraine. Severe, persistent headaches may respond well to nortriptyline (Pamelor) or another TCA. Among the TCAs, amitriptyline (Elavil) has been studied most extensively but nortriptyline (Pamelor), imipramine (Tofranil), protriptyline (Vivactil) and desipramine (Norpramine) are effective as well and may have fewer anticholinergic effects.

If one TCA is ineffective or produces unacceptable side effects, consider a trial with another. The starting dose for most TCAs is $25 \mathrm{mg}$ in a young or middle-aged patient and $10 \mathrm{mg}$ in an elderly person. The average effective dosage, however, is 50 to $75 \mathrm{mg}$ taken once a day in the evening. The patients must be told that these medications are antidepressants, but that they are also used as analgesics to treat chronic pain, even in the absence of depression. It is essential to explain this to patients so that they do not assume that the physician interpreted their symptoms as a manifestation of depression, rather than "real pain." Patients should also understand the possibility of side effects such as dry mouth, drowsiness, and constipation and take some preventive measures, especially against constipation. Contraindications to the use of TCAs include concomitant use of monoamine oxidase inhibitors, recent myocardial infarction, cardiac arrhythmias, glaucoma, and urinary retention. An ECG should be obtained before the initiation of treatment in all elderly patients or those with heart disease.

Selective serotonin re-uptake inhibitors (SSRIs), such as fluoxetine (Prozac), sertraline (Zoloft) and paroxetine (Paxil) have fewer side effects but lack the analgesic effect of TCAs. They can sometimes help indirectly, by reducing anxiety and depression. SSRIs do not cause weight gain but may cause sexual dysfunction, especially loss of libido. Effexor (venlafaxine) is a serotonin and norepinephrine reuptake inhibitor and appears to have better efficacy for migraine headaches than SSRIs. Its potential side effects include insomnia and GI symptoms.

Propranolol, nadolol and other betablockers are good prophylactic agents. The effective dose for propranolol can be as low as $40 \mathrm{mg}$ daily but is usually 80 to $240 \mathrm{mg}$. Contraindications include bronchial asthma, sinus bradycardia, greater than first degree block, congestive heart failure, and diabetes. Angiotensin II receptor antagonist, candesartan (Atacand) was shown to prevent migraines in a double-blind trial. Calcium channel blockers are not very effective for the treatment of migraines.

Divalproex sodium (Depakote, Depakote ER) relieves migraine headaches in patients who do not respond to betablockers or antidepressants. The starting dosage is usually $250 \mathrm{mg} / \mathrm{d}$ with a gradual increase to $2000 \mathrm{mg}$. Potential side effects include nausea, drowsiness, alopecia, and weight gain. Other anticonvulsants that can be useful in the prophylaxis of migraines are topiramate (Topamax), zonisamide (Zonegran), levetiracetam (Keppra) and gabapentin (Neurontin). Divalproex sodium is teratogenic, and women must observe strict contraceptive measures. Topiramate has the advantage of inducing weight loss, but cognitive side effects in some patients can be intolerable.

\section{Using Botox in the Treatment of Migraine}

Initially, the author limited the use of Botox to patients suffering from headaches that were highly refractory to drug and other therapies. A large minority of these patients showed significant and sustained improvement, often after many years of unrelieved pain and associated symptoms. This led to the use of Botox in patients suffering from intermittent migraine headaches who needed prophylactic therapy. The author's experience sug- gests that the group of patients most likely to benefit are those with intermittent migraine headaches, in whom he finds that Botox is effective (defined as at least a $50 \%$ improvement in frequency or severity) in $70 \%$ to $80 \%$ of patients (13), as well as those with chronic migraines, with or without rebound phenomenon. Less likely to respond are patients with chronic tension-type and cluster headaches. No other clinical predictors of response have been identified, although it is possible that patients with pericranial and neck tenderness, which may reflect more pronounced muscle spasm, are more likely to respond. The presence of temporomandibular joint tenderness (TMJ syndrome) also suggests hyperactivity of muscles.

\section{Injection Technique}

The sites injected usually include frontalis, glabellar, procerus, temporalis, masseter, splenius capiti, paraspinal, cervical, and trapezius muscles, depending on the distribution of migraine pain and trigger points found on palpation. The average dose for the treatment of headaches is 70 to 100 units. Higher amounts may be needed, especially in patients with pronounced associated spasm in masticatory, neck, trapezii, and other upper back muscles.

The usual dilution is $100 \mathrm{U}$ of Botox in $4 \mathrm{~mL}$ of normal saline, although some physicians dilute a vial in half that volume. More concentrated solutions and EMG-guidance are used for movement disorders because of the need for precise localization and paralysis of specific muscles. In the treatment of patients with headaches and myofascial pain syndromes, a somewhat wider distribution of muscle paralysis is desired. A lower concentration allows for easier distribution of BT over a larger number of muscles. EMG-guidance is not necessary for the treatment of headaches, and it only increases the cost and discomfort of treatment.

The 100-unit vial is vacuum-filled, and the absence of a vacuum during dilution suggests that the contents of the vial will be ineffective. This is a very rare occurrence; the vial should be exchanged for another. According to the product label, preservative-free saline should be used and the solution administered within 4 hours after dilution. However, saline with preservative possibly makes injections less painful (anecdotal observation) and al- 
lows diluted Botox to be used safely for several days after the dilution. The author injected his own frontalis muscle with Botox two weeks after dilution and experienced complete paralysis. Diluting Botox with $1 \%$ lidocaine or $0.5 \%$ bupivacaine has been reported without loss of efficacy of Botox. Using a local anesthetic provides immediate relief and could potentially reduce the initial worsening of pain seen in a small number of patients.

Myobloc is available only in a diluted form and only in a concentration of 5,000 units per milliliter. When used for the management of headaches and pain rather than movement disorders, further dilution is necessary. Myobloc solution has a very acidic $\mathrm{pH}$, which makes injections, especially in the forehead, significantly more painful.

The use of a 30-gauge, 1-inch needle is recommended. The incidence of cosmetic and other side effects usually declines with the increase in the experience of the injector. An additional small injection of Botox easily corrects any visible eyebrow asymmetry or pronounced unilateral wrinkling or exaggerated elevation of the lateral aspect of an eyebrow. Ptosis can sometimes be relieved by instilling $0.5 \%$ apraclonidine (Iopidine), alpha adrenergic agonist eye drops 3 times a day. On rare occasions, a patient will complain of the cosmetic effects of temporalis muscle wasting. If this if of significant concern to the patient, injections of the anterior temporalis muscle can be avoided on subsequent treatments.

Antibody formation can sometimes render BT ineffective. More frequent administration and higher doses increase the risk of antibody formation. The incidence of antibody formation is probably also affected by the protein load of the preparation. Therefore the Botox preparation of BT-A may be least likely to produce antibodies, because $100 \mathrm{U}$ of Botox contain only $5 \mathrm{ng}$ of protein, while the other 2 commercially available preparations have a significantly higher protein load. Patients who develop antibodies to one type of toxin may respond to another type. The author's anecdotal experience suggests that people who become unresponsive to BT often regain responsiveness after a period of about 6 months.

The development of antibodies to botulinum toxin is a rare complication that renders treatment ineffective. People immunized against BT in the military or at toxin manufacturing plants also do not develop muscle paralysis. If a patient fails to develop visible paralysis of the frontalis muscles, a brow furrow test can help differentiate antibody formation from a defective product. This test consists of injecting about $2 \mathrm{U}$ of Botox into the glabellar area. If the injection again fails to produce paralysis, the patient has developed antibodies.

Large trials are needed to provide unequivocal proof of efficacy, although it appears unlikely that Botox works largely through a placebo effect. We have encountered many patients who responded to Botox, but failed to demonstrate a placebo response to similarly dramatic treatments such as injections of other drugs, nerve blocks, or acupuncture. Also, the placebo effect typically does not last for more than a few months, while some of our patients have been responding to repeated treatments with Botox for up to 6 years. Anecdotal reports, as well as our experience, suggest that in some patients, repeated treatments with Botox provide increasing benefits with longer duration of effect and more complete relief with each subsequent treatment $(10,13)$. In some patients, a single treatment suffices to break the pattern of chronic headaches without the need for repeat injections, although simultaneous interventions such as withdrawal from abortive drugs and caffeine and lifestyle changes may also account for some of the improvement. Several questions about the proper use of Botox remain to be answered by large prospective trials that are currently underway. These include uncertainty about the optimal sites for and number of injections, the optimal dilution of Botox, the types of headaches that may respond to Botox, and the long-term efficacy of this agent.

\section{Long-Term Results}

Several clinicians report successful long-term use of BT in the treatment of headaches $(5,10,11)$. Retrospective review of more than 560 charts of our patients with headaches treated with Botox confirms not only the very benign side-effect profile of Botox but also its high efficacy in preventing migraine headaches. The author examined the long-term efficacy of this treatment by identifying 120 patients with intermittent and chronic migraines who have received 4 or more treatments with Botox. The average number of treatments was 6 (highest, 13) over a mean period of 15 months (range, 4 to 39 months). The average dose was $65 \mathrm{U}$ (range, 25 to 200). At the time of this review, we have treated more than 800 headache patients with Botox.

\section{SUMmaRY}

The author's general approach to the treatment of headaches begins with the elimination of headache triggers, the most common ones being caffeine in the diet and in medications such as Excedrin, Fioricet and Esgic. Nonpharmacological prophylactic approaches, such as regular aerobic exercise, biofeedback and nutritional supplements, including magnesium, feverfew, CoQ10, and riboflavin can be very effective in some patients.

For abortive therapy, triptans, which include rizatriptan (Maxalt), sumatriptan (Imitrex), almotriptan (Axert), zolmitrip$\tan$ (Zomig), eletriptan (Relpax), naratriptan (Amerge) and frovatriptan (Frova), should be used almost exclusively. If headaches remain frequent or refractory to treatment, prophylactic medications should be considered, despite their significant limitations.

While the FDA has approved only 4 drugs for migraine prophylaxis (methysergide, propranolol, timolol, divalproex sodium), many more are used for this purpose. Many of the unapproved drugs are marginally effective, while others often cause unacceptable side effects. When given descriptions of the potential side effects of medications such as propranolol, divalproex sodium, or amitriptyline many patients are reluctant to take them. BTA lacks systemic or other serious side effects, and its long-term safety has been established through its extensive use in the treatment of a variety of disorders over the past 20 years. In addition, compliance with a daily medication for some patients is difficult, while Botox injections are usually performed every 3 months.

BT treatment of headaches appears to be cost-effective (29). BT can often reduce costs by reducing the need for expensive triptans and other drugs and by the elimination of side effects associated with oral prophylactic agents, triptans, and other abortive drugs. While we await the results of large definitive studies the extensive experience of a large number of headache specialists in almost all ma- 
jor headache clinics in the United States, as well as multiple reports and reviews, suggest that BT is a simple, safe, effective and cost-effective treatment for headaches, especially for episodic and chronic migraines.

\begin{tabular}{l} 
Author Affiliation: \\
Alexander Mauskop, MD \\
Director \\
New York Headache Center \\
Associate Professor of \\
Clinical Neurology \\
SUNY-Downstate Medical Center \\
30 East 76th Street \\
New York, NY 10021 \\
E-mail: NYheadache@aol.com \\
\hline
\end{tabular}

\section{RefERENCES}

1. Erbguth FJ, Naumann, M. Historical aspects of botulinum toxin. Justinius Kerner (1786-1862) and the "sausage poison". Neurology 1999; 53:1850-1853.

2. Sloop RR, Cole BA, Escutin RO. Human response to botulinum toxin injection: Type B compared with type A. Neurology 1997; 49:189-194.

3. Jankovic J, Schwartz K. Botulinum toxin injections for cervical dystonia. Neurology 1990; 40:277-280.

4. Foster L, Clapp L, Erickson M et al. Botulinum toxin $\mathrm{A}$ and chronic low back pain. $\mathrm{A}$ randomized, double-blind study. Neurology 2001; 56:1290-1293.

5. Binder WJ, Brin MF, Blitzer A et al. Botulinum toxin type A (BOTOX) for treatment of migraine headaches: an open-label study. Otolaryngol Head Neck Surg 2000; 123: 669-676.

6. Giladi N. The mechanism of action of botulinum toxin type $A$ in focal dystonia is most probably through its dual effect on efferent (motor) and afferent pathways at the injected site. I Neurol Sci 1997; 152:132135.

7. Aoki KR. Evidence for antinociceptive activity of botulinum toxin type $A$ in pain management. Headache. 2003; 43:S9S15.

8. Göbel $\mathrm{H}$, Heinze A, Heinze-Kuhn $\mathrm{K}$ et al. Botulinum toxin $A$ in the treatment of headache syndromes and pericranial pain syndromes. Pain 2001; 91:195-199.

9. Göbel $\mathrm{H}$, Lindner $\mathrm{V}$, Krack $\mathrm{P}$ et al. Treatment of chronic tension-type headache with botulinum toxin [abstract]. Cephalalgia 1999; $19: 455$.

10. Smuts JA, Barnard PWA. Botulinum toxin type $A$ in the treatment of headache syndromes: a clinical report of 79 patients [abstract]. Cephalalgia 2000; 20:332.

11. Mauskop A, Basdeo R. Botulinum toxin $A$ is an effect prophylactic therapy of migraines [abstract]. Cephalalgia 2000; 20: 422.

12. Mauskop A. Long-term use of botulinum toxin type $A$ in the treatment of episodic and chronic migraine headaches [abstract]. Headache 2002; 42:454-455.

13. Mauskop A. The use of botulinum toxin in the treatment of headaches. Curr Pain Headache Rep 2002; 6:320-323.

14. Brin MF, Swope DM, O’Brian C et al. Botox for migraine: Double-bind, placebocontrolled region-specific evaluation [abstract]. Cephalalgia 2000; 20:421-422.

15. Krack P, Hornig C, Dorndorf W. Resolution of chronic tension headache after botulinum toxin treatment of idiopathic blepharospasm. Mov Disord 1995; 10:388.

16. Schulte-Mattler WJ, Wieser T et al. Treatment of tension-type headache with botulinum toxin: a pilot study. Eur J Med Res 1999; 4:183-186.

17. Relja MA. Treatment of tension-type headache with botulinum toxin: 1-year followup [abstract]. Cephalalgia 2000; 20:336.

18. Relja MA. Treatment of tension-type headache by injection of botulinum toxin type A: double blind placebo-controlled study. Eur J Neurol 1997; 4:S71-S73.

19. Relja MA, Klepac N. Botulinum toxin type $A$ as a prophylactic treatment for chronic tension type headache: long term followup study [abstract]. Neurology 2001; 56: A203.

20. Rollnik JD, Tanneberger O, Schubert $M$ et al. Treatment of tension-type headache with botulinum toxin type $A$ : A doubleblind, placebo-controlled study. Headache 2000; 40:300-305.

21. Freund BJ, Schwartz M. Treatment of chronic cervical-associated headache with botulinum toxin A: A pilot study. Headache 2000; 40:231-236.

22. Hobson DE, Gladish DF. Botulinum toxin injection for cervicogenic headache. Headache 1997; 37:253-255.

23. Klapper JA, Mathew NT, Klapper A et al. Botulinum toxin type A (BT-A) for the prophylaxis of chronic daily headache [abstract]. Cephalalgia 2000; 20:291-292.

24. Klapper JA, Klapper A. Use of botulinum toxin in chronic daily headaches associated with migraine. Headache Quart 1999; 10:141-143.

25. Klapper J, Mathew N. Botulinum toxin type A (BTX-A) for the prophylaxis of chronic daily headache [abstract]. Cephalalgia 2000; 20:292-293.

26. Freund $B$, Schwartz M. The use of botulinum toxin $A$ in the treatment of refractory cluster headache: case reports [abstract]. Cephalalgia 2000; 20:329-330.

27. Ginies PR, Fraimout JL, Kong A et al. Treatment of cluster headache by subcutaneous injection of botulinum toxin. Program and abstracts of the 8th World Congress on Pain. 1996. Abstract 50.

28. Silberstein S, Mathew N, Saper J et al. Botulinum toxin type $\mathrm{A}$ as a migraine preventive treatment. Headache 2000; 40:445450.

29. Blumenfeld AM. Impact of botulinum toxin type $A$ treatment on medication costs and usage in difficult-to- treat chronic headache: case studies. Headache Quar 2001; 12:241-244.

30. Relja MA. Botulinum toxin type-A reduces acute medication (triptans) use in migraine patients [abstract]. Neurology 2003; 60:147.

31. Poungvarin $\mathrm{N}$. The first world report of botulinum $A$ toxin injection for status migrainosus. J Med Assoc Thai 2001; 84:11991203.

32. Blumenfeld $A$. Botulinum toxin type $A$ as an effective preventive treatment in headache [abstract]. Headache 2003; 43:576.

33. Mathew N, Kaup A, Kailasam J. Botulinum toxin type A modifies chronic migraine; further long-term (3 years) experience with up to ten sets of treatments [abstract]. Headache 2003; 43:576.

34. Barrientos N, Chana P. Efficacy and safety of botulinum toxin type $A$ (BOTOX) in the prophylactic treatment of migraine [abstract]. Headache 2002; 42:452.

35. Eross EJ, Dodick DW. The effects of botulinum toxin type $A$ on disability in episodic and chronic migraine [abstract]. Neurology 2002; 58:A497.

36. Krusz J. Intradermal botulinum toxin, type $\mathrm{A}$, for cervicogenic migraine [abstract]. Headache 2002; 42:405.

37. Smuts JA, Baker MK, Smuts HM et al. Prophylactic treatment of chronic tensiontype headache using botulinum toxin type A. Eur J Neurol 1999; 6:S99-S102.

38. Freund BJ, Schwartz M. Treatment of whiplash associated neck pain [corrected] with botulinum toxin-A: a pilot study. J Rheumatol 2000; 27:481-484.

39. Porta M. A comparative trial of botulinum toxin type $A$ and methylprednisolone for the treatment of tension-type headache. Curr Rev Pain 2000; 4:31-35.

40. McAllister P. Patient-reported improvements in headache and change in headache medication usage in a cohort treated with botulinum toxin type-A (Botox) [abstract]. Headache 2003; 43:577.

41. Tepper SJ, Bigal ME, Sheftell FD et al. The effect of botulinum toxin on the disability of refractory headaches [abstract]. Headache 2003; 43:578.

42. Miller T, Denny L. Botulinum toxin A (Allergan) for chronic intractable headache: equally effective with or without concomitant neck pain [abstract]. Headache 2003; 43:579.

43. Tomosovic J, Sabo T. Improvement of intractable headache in adolescent females after craniocervical injections of BTX-A (Allergan) [abstract]. Headache. 2003; 43: 579.

44. Loder E. Botulinum toxin treatment of headache and neck pain associated with stiff-person syndrome [abstract]. Headache 2003; 43:509.

45. Troost T, Rosenberg JR, Wiles R. Improvement in intractable headache with repeat- 
ed botulinum toxin type $A$ treatment [abstract]. Neurology 2003;60:A323-A324.

46. Wheeler $A H$. Botulinum toxin $A$, adjunctive therapy for refractory headaches associated with pericranial muscle tension. Headache 1998; 38:468-471.

47. Sebastian FT, de Bruijn M. Treatment of chronic tension-type headache with botulinum toxin; a double-blind placebo controlled clinical trial [abstract]. Neurology 2003; 60:148.

48. Blumenfeld AM. Botulinum toxin type $A$ (BOTOX®) as an effective prophylactic treatment in headache [abstract]. Cephalalgia 2002; 22:20.

49. Troost BT. Botulinum toxin type A (BOTOX) therapy for intractable headache [abstract]. Headache 2002; 42:435-436.

50. Padberg M, De Bruijn SJ. Treatment of chronic tension-type headache with botulinum toxin: A double-blind, placebocontrolled clinical trial [abstract]. Neurol 2002; 1:63.

51. Ondo WG, Derman HS. Botulinum toxin type $A\left(\right.$ BOTOX $\left.^{\circledR}\right)$ for chronic daily headache: A 60 subject, randomized, placebo-controlled, parallel design study [abstract]. Headache 2002; 42;431.

52. Lopez-Lozano LL. Clinical study of the value of botulinum toxin-A (Botox) in the treatment of chronic tension-type headache with associated pericranial muscle tenderness [abstract]. Cephalalgia 2002; 22:32-33.

53. Lin JJ, Chang DC. Efficient treatment of chronic tension-type headache with botulinum toxin-A [abstract]. Cephalalgia. 2002; 22:15-16.

54. Miller T, Denny L. Retrospective cohort analysis of 48 chronic headache patients treated with botulinum toxin type A (BOTOX) in a combination fixed-injection -site and "follow the pain" protocol [abstract]. Headache 2002; 42:430-431.

55. Edwards KR. Botulinum toxin type A (Botox) for chronic daily headaches [abstract]. Headache 2002; 42:429.

56. Freund BJ, Schwartz M. Treatment of whiplash associated neck pain with botulinum toxin-A: report of 8 cases. / Rheumatol
1999; 26:756-775.

57. Wollina U. Botulinum A toxin for wrinkles: release from tension headache. J Eur Acad Dermatol Venereol 2000; 14:142-143.

58. Johnstone SJ, Adler $\mathrm{CH}$. Headache and facial pain responsive to botulinum toxin: an unusual presentation of blepharospasm. Headache 1998; 38:366-368.

59. Silberstein SD. Review of botulinum toxin type $A$ and its clinical applications in migraine headache. Expert Opin Pharmacother 2001; 2:1649-1654.

6o. Royal MA. The use of botulinum toxins in the management of pain and headache. Pain Pract 2001; 1:215-235.

61. Mathew NT; Kaup AO. The use of botulinum toxin type $A$ in headache treatment Curr Treat Options Neurol 2002; 4:365373.

62. Chapman SL. A review and clinical perspective on the use of EMG and thermal biofeedback for chronic headaches. Pain 1986; 27:1-43.

63. Blanchard EB. Long term effects of behavioral treatment or chronic headache. Behav Ther 1987; 8:375-385.

64. Gauthier JG, Carrier S. Long-term effects of biofeedback on migraine headache: A prospective follow-up study. Headache 1991; 31:605-612.

65. Mauskop A, Altura BT, Cracco RQ et al. Intravenous magnesium sulfate relieves $\mathrm{mi}$ graine attacks in patients with low serum ionized magnesium levels: a pilot study. Clin Science 1995; 89:633-636.

66. Facchinetti F, Sances G, Borella P et al. Magnesium prophylaxis of menstrual migraine: effects on intracellular magnesium. Headache 1991; 31:298-301.

67. Peikert A, Wilimzig C, Kohne-Volland R. Prophylaxis of migraine with oral magnesium: results from a prospective, multicenter, placebo-controlled and doubleblind randomized study. Cephalalgia 1996; 16:257-263.

68. Pfaffenrath V, Wessely P, Meyer C, et al. Magnesium in the prophylaxis of migraine: a double-blind, placebo-controlled study. Cephalalgia 1996; 16:436-440.
69. Schoenen J, Jacquy J, Lenaerts M. Effectiveness of high-dose riboflavin in migraine prophylaxis. Neurology 1998; 50 : 466-470.

70. Sandor PS, Di Clemente L Coppola G et al. Coenzyme Q10 for migraine prophylaxis: a randomized trial [abstract]. Cephalalgia 2003; 23:577.

71. Vogler BK, Pittler MH, Ernst E. Feverfew as a preventive treatment for migraine: a systematic review. Cephalagia 1998; 18:704708.

72. Murphy JJ, Heptinsall S, Mitchell JRA. Randomized double-blind placebo-controlled trial of feverfew in migraine prevention. Lancet 1988; 2:189-192.

73. Pfaffenrath $V$, Diener $\mathrm{HC}$, Fischer $\mathrm{M}$ et al. The efficacy and safety of Tanacetum parthenium (feverfew) in migraine prophylaxis--a double-blind, multicentre, randomized placebo-controlled dose-response study. Cephalalgia 2002; 22:523532.

74. Grossman M, Schmidramsl H. An extract of Petasites hybridus is effective in the prophylaxis of migraine. Int I Clin Pharmacol Ther 2000; 38:430-435.

75. Lipton RB, Gobel H, Wilks K et al. Efficacy of petasites (an extract from petasites rhizone) 50 and $75 \mathrm{mg}$ for prophylaxis of migraine: results of a randomized, doubleblind, placebo-controlled study. Neurology 2002; 58:A472.

76. Han JS, Terenius L. Neurochemical basis of acupuncture analgesia. Ann Rev Pharmacol Toxicol 1982; 22:193-220.

77. Melchart D, Linde K, Fischer P, et al. Acupuncture for recurrent headaches: a systematic review of randomized controlled trials. Cephalalgia 1999; 19:779-786.

78. Ferrari MD, Roon KI, Lipton RB et al. Oral triptans in acute migraine treatment: A meta-analysis of 53 trials. Lancet 2001; 358:1668-1675.

79. Mauskop A, Altura BT, Cracco RQ et al. Deficiency in serum ionized $\mathrm{Mg}$ but not total $\mathrm{Mg}$ in patients with migraine. Possible role of $\mathrm{ICa}^{2+} / \mathrm{IMg}^{2+}$ ratio. Headache 1993; 33: 135-138. 
